doi: http://dx.doi.org/10.7124/bc.0009F4

\section{R-1. Structural studies of PML nuclear bodies in polyomavirus infected cells}

Boris Ryabchenko and Jitka Forstova

Charles University, Faculty of Science, Biocev, Prague, Czech Republic

ryabcheb@natur.cuni.cz

ProMyelocytic Leukemia Nuclear Bodies (PML NBs) are distinct dynamic nuclear substructures (approx. 1 micron in diameter) implicated in different physiological and pathological cellular processes, including virus infection. While large viruses, e.g. herpesviruses cause their disruption, smaller DNA viruses, as papilloma- or polyomaviruses, realize parts of the reproduction cycle in their close proximity. Previously, we found that Mouse polyomavirus (MPyV) infection causes multiplication and enlargement of PML NBs. During late phases of infection, the integrity and morphology of PML NBs are visibly altered. In addition, we observed the accumulation of MPyV virions around and inside of PML NBs. The aims of our research are: 1 . To find whether replication of MPyV genomes itself or rather assembly of virions is responsible for altering the integrity and morphology of PML NBs. 2. To reveal the process of multiplication of PML NBs in infected cells. 3. To visualize the interaction of viral structural and regulatory proteins with PML NBs. We found that replication of mutated MPyV, capable of genome replication and production of all regulatory proteins, is sufficient to alter the morphology of PML NBs, althought it lacks ability to produce structural proteins. Live cell microscopy revealed that in infected cells, PML NBs are highly dynamic structures that assemble from soluble PML NBs' proteins as well as by fusion or fission of pre-existing nuclear bodies. Using structured illumination microscopy (SIM) and stochastic optical reconstruction microscopy (STORM), we observed the major structural protein of MPyV VP1, - to be located inside PML NBs, while the regulatory large $T$ antigen ( bound to replicating MPyV genomes) was located by the surface of PML NBs.

doi: http://dx.doi.org/10.7124/bc.0009F5

\section{S-1. The dispersal of ribosomal gene sequences in the karyotype of Coturnix japonica}

Alsu Saifitdinova ${ }^{1,2}$, Svetlana Galkina ${ }^{3}$, Maria Kulak ${ }^{3}$, Valérie Fillon, Valeria Volodkina $^{3}$, Olga Pavlova ${ }^{2,5}$, Elena Gaginskaya ${ }^{3}$

${ }^{1}$ Herzen State Pedagogical University of Russia, Saint Petersburg, Russia; ${ }^{2}$ International Centre of Reproductive Medicine, Saint Petersburg, Russia; ${ }^{3}$ Saint Petersburg State University, Saint Petersburg, Russia; ${ }^{4}$ National Institute of Agricultural Research, Toulouse, France; ${ }^{5}$ Beagle Ltd., Saint Petersburg, Russia saifitdinova@mail.ru

Numerous copies of rDNA genes form nucleolar organizer regions (NORs) on chromosome and give rise to the interphase nucleoli. Bird species commonly have a single pair of the NOR bearing chromosomes in the karyotype (Schmid et al., 1982). Japanese quail is an exception and has three pairs of chromosomes with active NOR (McPherson et al., 2014). 
Even within a single NOR array individual sequences may differ (Kim et al., 2018). This work was aimed at studying the individual sequences that make up different NORs in Coturnix japonica. Methods: Using primers to the conserved region of the $18 \mathrm{~S}$ ribosomal RNA gene, we amplified rDNA the range of fragments from the quail genome karyotype. The rDNA fragments were cloned, sequenced, analyzed bioinformatically and mapped by FISH. Results: Ribosomal gene derivatives were found to localize on the short heterochromatic arms of all acrocentric chromosomes in the complement. In addition to functional NOR sequences, we have found chimeric sequences containing fragments of transposable elements, fragments of MHC genes and some others. As it was shown earlier active transcription makes NOR a target for transposons and causes mutual amplification of the chimeric sequences of transposons and ribosomal genes (Buzdin et al., 2007). Conclusions: In the genome of Japanese quail transposition of NOR sequences apparently led to the dispersal and amplification of NORs, three of which retained their functionality.

Financial and technical support: RFBR \#18-04-01276-a, Research Resource Centres "Chromas" and "Molecular and Cell Technologies".

References: Schmid M, Loser C, Schmidtke J, Engel W. Evolutionary conservation of a common pattern of activity of nucleolus organizers during spermatogenesis in vertebrates. Chromosoma, 1982. 86:149-179. McPherson MC, Robinson CM, Gehlen LP, Delany ME. Comparative cytogenomics of poultry: mapping of single gene and repeat loci in the Japanese quail (Coturnix japonica). Chromosome Res. 2014. 22:71-83. Kim JH, Dilthey AT, Nagaraja R, Lee HS, Koren S, Dudekula D, Wood III WH, Piao Y, Ogurtsov AY, Utani K, Noskov VN, Shabalina SA, Schlessinger D, Phillippy AM, Larionov V. Variation in human chromosome 21 ribosomal RNA genes characterized by TAR cloning and long-read sequencing. NAR, 2018. 46:6712-6725. Buzdin A, Gogvadze E, Lebrun M-H. Chimeric retrogenes suggest a role for the nucleolus in LINE amplification. FEBS Letters, 2007. 581:2877-2882.

doi: http://dx.doi.org/10.7124/bc.0009F6

\section{S-2. Role of Epstein-Barr Virus Zebra protein in induction of $t(8 ; 14)$ translocation}

Fatimata B. Sall ${ }^{1,2}$, Diego Germini ${ }^{1}$, Anna Shmakova $^{3}$, Papa Malick D. Diouf ${ }^{4}$, Joëlle Wiels ${ }^{1}$, Moussa Ndour ${ }^{5}$, Awa O. Touré ${ }^{2}$, Yegor Vassetzky ${ }^{1}$

${ }^{1}$ UMR8126 CNRS, Paris-Sud University, Gustave Roussy Institute, Villejuif, France; ${ }^{2}$ Hematology Laboratory, Dantec Hospital Center, Cheikh Anta Diop University, Dakar, Senegal; ${ }^{3}$ Laboratory of Gene and Cell Technologies, Faculty of Medicine, Lomonosov Moscow State University, Moscow, Russia; ${ }^{4}$ Pediatric Department, Dantec Hospital Center Dakar, Senegal; ${ }^{5}$ Laboratory of Bacteriology and Virology, Dantec Hospital Center, Dakar, Senegal

fabisall3007@gmail.com

Burkitt lymphoma (BL) is an aggressive NonHodgkin Lymphoma characterized by a chromosomal translocation involving the MYC oncogene located on the chromosome 8 and one of the immunoglobulin gene loci located on the chromosomes 2, 14 or 22. The African endemic form of $\mathrm{BL}$ is associated with the Epstein Barr Virus (EBV). EBV life cycle includes two phases: lytic and latent. The switch from latency to lytic cycle (EBV reactivation) is initiated by the EBV Zebra protein. We study the role of EBV reactivation in the formation of one BL characteristic translocation $\mathrm{t}(8 ; 14)$. Our hypothesis is that the Zebra- 